\title{
New Fitting Concept in ATLAS muon tracking for the LHC Run-2
}

\author{
William Leight ${ }^{1, *}$, Pierre-Francois Giraud ${ }^{2, * *}$, Peter Kluit ${ }^{3, * * *}$, Jochen Meyer ${ }^{4, * * * *}$,Edward \\ Moyse $^{5, \dagger}$, and Alan Poppleton ${ }^{6, \ddagger}$ on behalf of ATLAS Computing \\ ${ }^{1}$ Deutsches Elektronen-Synchrotron DESY, Hamburg and Zeuthen, Germany \\ ${ }^{2}$ IRFU, CEA, Université Paris-Saclay, Gif-sur-Yvette, France \\ ${ }^{3}$ Nikhef National Institute for Subatomic Physics and University of Amsterdam, Amsterdam, Nether- \\ lands \\ ${ }^{4}$ Department of Physics, Technion, Israel Institute of Technology, Haifa, Israel \\ ${ }^{5}$ Department of Physics, University of Massachussetts, Amherst, MA, USA \\ ${ }^{6} \mathrm{CERN}$, Geneva, Switzerland
}

\begin{abstract}
Muons with high momentum - above $500 \mathrm{GeV}$ - are an important constituent of new physics signatures in many models. Run-2 of the LHC is greatly increasing ATLAS's sensitivity to such signatures thanks to an everlarger dataset of such particles, enhanced further by an increase in the center-ofmass energy. The ATLAS Muon Spectrometer chamber alignment contributes significantly to the uncertainty of the reconstruction of these high-momentum objects. The proper treatment of measurements during tracking and the correct propagation of the alignment effects is extremely challenging. Recently, an innovative approach that imposes Gaussian constraints on ensembles of detector hits was implemented. It provides a significant improvement to highmomentum tracking without increasing the CPU budget. Furthermore, it allows for the verification of the expected alignment quality using high-statistics collision data. A detailed discussion of the algorithmic realization is given, the expected performance gains are presented and prospects for further applications of the approach are outlined.
\end{abstract}

\section{Introduction}

Between the LHC Run-1 and Run-2, the accelerator's center-of-mass energy was increased from 8 to $13 \mathrm{TeV}$, allowing it to probe for new particles at ever higher masses, as suggested by a number of proposed extensions to the Standard Model. Final states in which such particles decay to muons are favorable for searches, as they are relatively clean with well-understood backgrounds. Naturally, the decay of particles with masses of multiple $\mathrm{TeV}$ will produce muons with very high momenta, making the accurate reconstruction of muons with momenta

\footnotetext{
*e-mail: william.leight@desy.de

**e-mail: pierre-francois.giraud@cern.ch

***e-mail: s01@nikhef.nl

****e-mail: jochen.meyer@cern.ch

†e-mail: edward.moyse@cern.ch

†e-mail: alan.poppleton@cern.ch
} 
of $500 \mathrm{GeV}$ or more essential for related searches for new particles. At such momenta, the precision with which the muon's momentum can be measured will be strongly affected by the alignment of the chambers in the Muon Spectrometer (MS): that is, by how much the real position of the chambers differs from the "perfect" detector assumed by the reconstruction software. For Run-2, a new method of handling misalignments, using Gaussian constraints on ensembles of detector hits, was implemented, resulting in improved reconstruction of muons overall and high-momentum muons in particular. Below, we describe briefly the reconstruction of muons in ATLAS and the measurement of the MS alignment parameters, and then how this new method uses the latter to improve the former.

\section{Muon reconstruction in the ATLAS detector}

The ATLAS detector is an all-purpose particle reconstruction detector at the LHC [1]. In the MS [2], a cross-section of which is shown in Figure 1a, precision measurements in the bending plane are provided over most of the range of $|\eta|<2.7$ by Monitored Drift Tube (MDT) chambers: at the highest values of $|\eta|$ in the innermost endcap layers, Cathode Strip Chambers (CSC) are used instead, as the flux is too high for MDTs. Full $\phi$ coverage is provided by overlapping chambers, alternately small or large as can be seen in Figure $1 b$. Bending in the MS is provided by toroidal magnetic fields. Muon tracking and reconstruction is performed using ATLAS's Athena software package. Tracking is first carried out separately in the MS and the Inner Detector (ID) using a fitting program based on $\chi^{2}$ minimization [3]. MS tracks that can be successfully extrapolated to the interaction point are then matched to ID tracks and a combined fit of all the measurements included in the two tracks is performed using a $\chi^{2}$ minimization which also takes into account energy loss in the calorimeters. During both the extrapolation and combined fit stages, MS hits can be added to or removed from the track. As can be seen in Figure 2, at low transverse momentum $\left(p_{T}\right)$, the resolution is dominated by energy loss and multiple scattering, but at higher values, where the amount of bending of the trajectory is minimal, precise knowledge of the alignment of the detector becomes more important. In particular, to achieve the intended design resolution of $10 \%$ at 1 $\mathrm{TeV}$ the position of the MS chambers must be known to better than $40 \mu \mathrm{m}$.

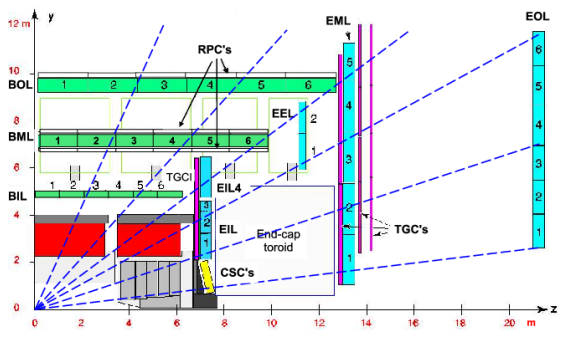

(a)

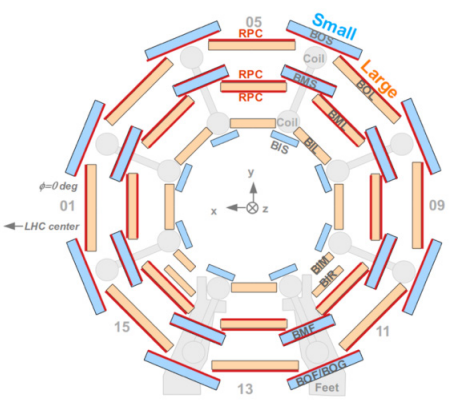

(b)

Figure 1: A cross-sectional view of the ATLAS detector, with parts of the MS identified (a) and a view of the MS along the $\mathrm{z}$ axis (b) [1]. 


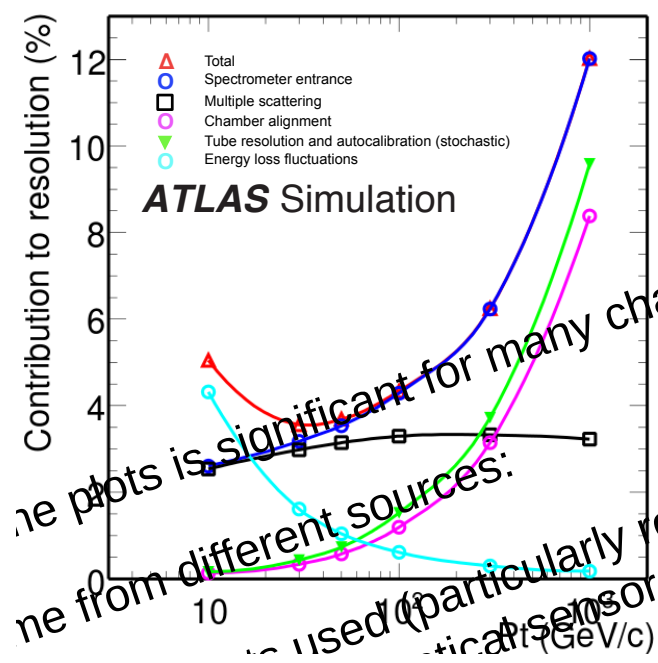

Figure 2: Contributions to the measured $p_{\mathrm{T}}$ resolution of muons vs. muon $p_{\mathrm{T}}[2]$

\section{Muon Spectrometer Alignment}

Track reconstruction is performed based on measurements, usually referred to as hits, i.e. on signals in given elements of the detector indicating the passage of a muon. During reconstruction, the locations of the detector elements in question are obtained from the muon tracking geometry, which is an idealized representation of where all the chambers in the detector are supposed to be. However, due to various factors the chambers may not in fact be in the position, and with the orientation, that the tracking geometry provides. Therefore it is necessary to cross-check this ideal geometry against the chambers' actual measured physical positions.

At the time of installation the positions of all the MS chambers were surveyed, but the survey accuracy was only a few $\mathrm{mm}$ : furthermore, the chambers are expected to move and/or deform over time. Therefore it is necessary to obtain the alignments of the chambers in situ: this is done using an optical alignment system [4], which measures the chamber position relative to the initial survey. In the barrel, three-point straightness monitors known as RASNIKs, consisting of a mask, a lens, and a CCD, are used: when the items are placed on different chambers, their relative alignment can be quantified. In the endcaps, where projective lines are not possible due to the presence of the endcap toroid magnets, a system of alignment bars, precision reference rulers, are used, with their alignments relative to each other and to the chambers monitored using BCAMs, in which a camera observes the position of a laser diode. These systems measure displacements and deformations of the chambers in various directions in order to constrain their positions and rotations.

However, the optical alignment alone is insufficient to achieve the desired resolution. Partly this is simply due to the fact that the location of the alignment sensors can only be known to the precision with which they were mounted, which imposes a constraint on the entire optical alignment. The optical alignment is also limited by the fact that it was impossible to create a single system that covered the whole of ATLAS. As a result, some chambers in the MS are only partly integrated into the system, the alignment systems of the small and large chambers are only partially connected, and the alignment systems for the barrel and endcap are not connected to each other (see Figure 3). Additionally, the alignments of the MS and ID are totally unconnected. Therefore, the optical alignment is supplemented by 
an additional track-based alignment, measured using tracks taken during runs in which the toroidal magnetic field is turned off. With no magnetic field, all tracks should be straight: deviations from straightness therefore represent misalignments. A sample of a few million tracks is used to perform the alignment, though it is still statistics-limited. A new alignment must be performed if the previous one is disturbed, by opening a chamber for repair, for instance. The results of the optical and track-based alignments are then combined to yield the total amount of misalignment $\mu$ of each chamber using the model:

$$
\mu_{0}+\frac{\theta-<\theta>}{R M S(\theta)} \mu_{\theta}+\frac{\phi-<\phi>}{R M S(\phi)} \mu_{\phi}
$$

This separates out the mean sagitta bias $\mu_{0}$ from the components of the misalignment along the polar $(\theta)$ and azimuthal $(\phi)$ angles: the RMS is calculated over the track sample.

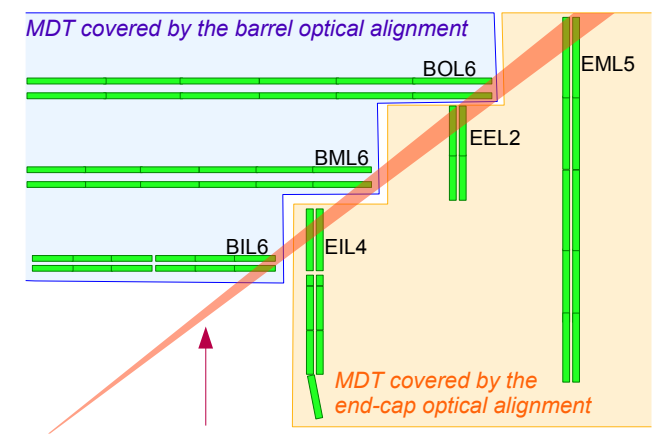

Figure 3: Illustration of the areas covered by the separate barrel and endcap optical alignment systems. The red shaded area indicates possible trajectories for straight-line tracks used in the tracking alignment to align the systems with respect to each other.

\section{Incorporating Alignment Effects in the Track-fitting Procedure}

Should the alignment measurements reveal that the real position and/or orientation of a given detector element is different from what is assumed in the tracking geometry, the track fit must be adjusted accordingly, including the accurate propagation of related uncertainties. Previously, this was accomplished by separately inflating the uncertainties assigned to hits in poorly aligned chambers. While this procedure made it possible for hits to be added to tracks even though their real position did not correspond to where the tracking geometry thought they should be, it came with significant drawbacks. First, as correlations were neglected, the uncertainty of the final $\chi^{2}$ fit did not reflect reality, and so could not be used to evaluate the quality of the fit. Additionally, as uncertainties were assigned to individual hits, the fit could move each hit to where it fit best, whereas in reality a misalignment will cause all the hits in a given chamber to move together. To overcome these flaws, a new method, using Gaussian constraints applied to ensembles of hits, was developed.

The width of the constraints are obtained from the actual misalignment measurements, with nominal values in the case where little to no misalignment is observed. Then each hit in a given chamber, or, for the case of tracks that contain hits from parts of the detector with separate alignments (tracks that overlap between the barrel and endcap, or between large and small sectors), each set of hits from multiple chambers, is given the same constraints. This 
ensures that when the hits move, they only move jointly. Further, uncertainties arising from alignment are properly correlated while the intrinsic hit uncertainties (due to e.g. detector resolution) are not touched, so the uncertainty on the final track is a meaningful quantity.

Implementation in the tracking is carried out using an event data model object known as an Alignment Effect on Track (AEOT). This object holds the list of hits it affects and the standard deviations of the constraints (angular or sagitta-based) it applies to the hits. Following the preliminary track fit, a refitting algorithm (the MuonRefitTool) constructs the appropriate AEOTs for the hits used in the track, based on misalignment information stored in a conditions database and processed by a specialized tool (the AlignmentErrorTool), and adds them to the vector of measurements that defines the track. The track refit is then performed, with the AEOTs included in the derivate matrix that enters the computation of the fit $\chi^{2}$, so that their effect is directly incorporated into the minimization process. Figure 4 shows the schematic workflow of the implementation in the code.

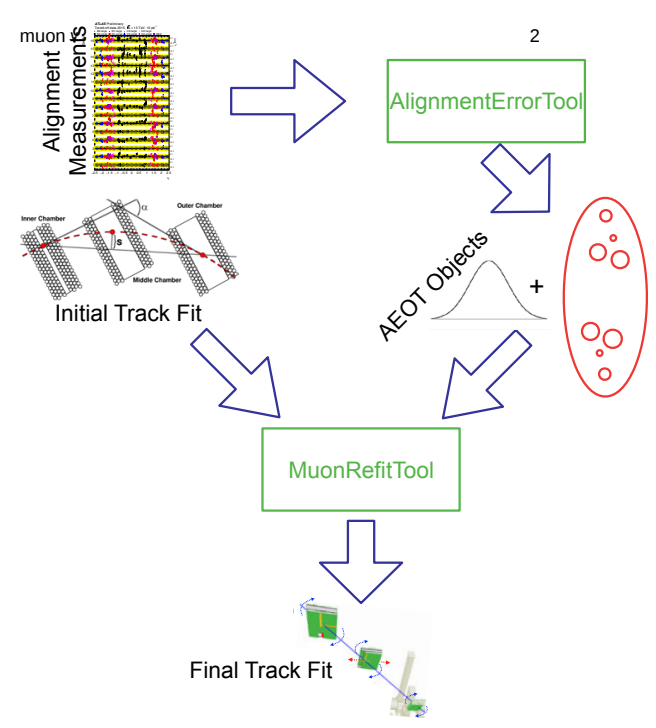

Figure 4: Sketch showing the code flow and tools used to construct the final track from the initial fit and the misalignment measurements described in Section 3.

\section{Results and Future Work}

A considerable improvement in the momentum resolution of reconstructed muons is observed thanks to these improvements. Figure 5a shows the dimuon mass resolution in simulated $Z$ boson decays obtained using Athena release 20.7 (with individual hit uncertainty inflation to account for misalignments) and 21.0 (in which AEOTs were implemented): the big improvement in the resolution in the barrel-endcap overlap region near $|\eta|=1$, where misalignment plays an especially important role due to the lack of connection between the barrel and endcap alignment systems, shows the power of the method. Figure $5 b$ shows the change in $q / p$ resolution (the ratio of charge to momentum, essentially the inverse momentum, is used to parameterize the track fit rather than the momentum itself) for higher-momentum tracks: as expected, the increasing importance of misalignment to the resolution as the $p_{\mathrm{T}}$ increases means that the AEOT method delivers performance improvements that increase with $p_{\mathrm{T}}$. 


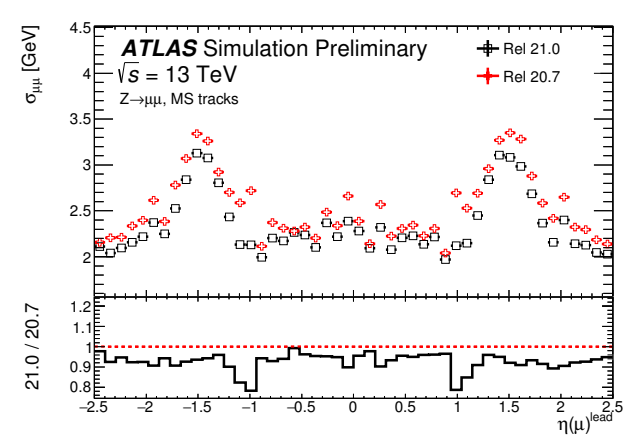

(a)

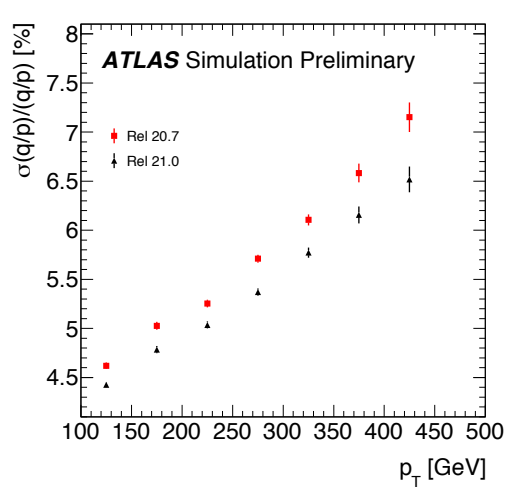

(b)

Figure 5: (a) Di-muon mass resolution from simulated $Z \rightarrow \mu \mu$ events plotted against the $\eta$ of the leading muon; (b) q/p resolution of muons reconstructed in simulated $Z \rightarrow \mu \mu$ events plotted vs. muon $p_{\mathrm{T}}[6]$.

In addition to the resolution improvements, the new method makes it possible, as mentioned, to evaluate the quality of the track fit using the fit uncertainty. This makes it possible for physics analyses to reject poor-quality muons directly, rather than applying geometric vetoes, increasing their muon selection efficiency.

While this is a significant gain, there is still room for further improvement. As previously discussed, the alignment systems of the ID and MS are completely separate. However, the current AEOT implementation does not attempt to address this issue. Instead, the degree of misalignment between the two detector subsystems is treated as a constant average value and implemented as an artificial scatterer without radiation length, allowing the track to change direction if necessary without losing energy. Expanding the AEOT implementation to handle this misalignment as well would allow for superior track reconstruction by greatly improving the granularity with which the misalignment is described.

Finally, as increased luminosity leads to more interactions per LHC bunch crossing and so an increase in the number of tracks that must be reconstructed in every event, we must remain cognizant of any CPU penalties. In this case, CPU consumption increased, as the matrix to be inverted for the fit became larger, but this increase was compensated for by migrating to the linear algebra package Eigen [5], leaving total CPU consumption roughly unchanged. However, if the use of AEOTs is to be further expanded, it may be necessary to rewrite the code to keep any additional CPU consumption to a minimum.

\section{References}

[1] ATLAS Collaboration, JINST 3 S08003 (2008).

[2] ATLAS Collaboration, ATLAS muon spectrometer: Technical Design Report, (CERN, Geneva, 1997)

[3] Cornelissen, T.G., et al., Journal of Physics: Conference Series 1193 (2008)

[4] Amelung, C., Eur. Phys. J C 33 (Suppl 1) (2004)

[5] Eigen project, "Eigen" [software], version 3.3.5 (2018). Available from https://bitbucket.org/eigen/eigen/ (accessed 2018-09-21) 
[6] ATLAS Collaboration, Momentum resolution improvements with the inclusion of the Alignment Errors On Track, https://atlas.web.cern.ch/Atlas/GROUPS/ PHYSICS/PLOTS/MUON-2018-003/ 